\title{
EL HOLISMO, UN NUEVO PARADIGMA DE LA CIENCIA ENFERMERA
}

Lic Sofia Rodriquez limenez" y Mtra Sandra Sotomayor Sanchez.".

- Profesora de Carrero Titular A. Titular del Cloustro de Teoria y Método de Enfermería. ENEO-UNAM,

"Profesora de Carrera Asociodo B. Presidenta de la Acodemia de Enfermeria Fundamental, ENEO-UMNAM

El conocimiento de la Enfermería requiere hoy en día, de una clara definición en sus teorias y conceptos que fundamenten la ciencia del cuidado, tanto para la investigación, la formación y la práctica. Este trabajo propone analizar el cuidado holístico como paradigma cientifico, lo cual tiene gran importancia para la construcción disciplinar y la consolidación de la identidad profesional.

El holismo se inscribe en el paradigma de la fenomenología critica por las caracteristicas de las ciencias humanistico sociales, esta consideración permite dar respuesta a nuevas interpretaciones de la realidad, se parte de la consideración de que la persona es un todo integrado, al reconocerle dimensiones psicosociales y culturales, biofisiológicas y espirituales. El otorgar cuidado holistico en la actualidad, constituye en si un compromiso ético profesional impostergable que las Enfermeras deberán considerar como parte fundamental para la formación académica y la práctica profesional.

La fenomenologla crítica como paradigma, privilegia la investigación integral, en donde se valora la comprensión de los lenguajes de las personas, grupos y comunidades, los significados que le asignan a sus experiencias de salud y de vida y para ello es necesario indagar en las creencias y valores de cada grupo social y poder construir el constructo "cuidado humano de la Enfermeria": Es meta deseable que las Enfermeras incursionen en la investigación de este fenómeno que logre rescatar los valores que caracterizan la cultura de los colectivos humanos en sus contextos especlficos y que les han permitido resolver problemas de salud, y que son relevantes en la fundamentación de la naturaleza del cuidado.

PALABRAS CLAVES: Enfermeria, Holismo, Cuidado holistico, paradigma, hermenéutica, fenomenología critica..

\section{Abstract}

The knowledge of nursing nowadays requires of a clear definition in its concepts and theories that base the saience of nursing care not only for research, the forming and the practice. This work suggests analyzing Holistic care as a scientific paradigm, which has a great importance for disciplinary construction and the consolidation of a professional identity.

Holism is implied in the critical phenomenology paradigm due to the characteristics of humanistic sacial sciences, this consideration allows to get answers to new interpretations of reality, it parts from the consideration af a person as an integrated whole, as an integrated total, when recognizing psychosocial, cultural, biophysiological and spiritual dimensions. 
Giving holistic care currently, constitutes a professional ethical commitment that cannot be postponed. This must be considered by the nurses as a fundamental part in their training and professional practice.

Critical phenomenology os a paradigm, privileges integral research, in which the understanding of the person's languages, groups and communities, the meaning they give to their health, and life experiences; for this, it is necessary to investigate the values and beliefs of each social group and be able to construct the "nursing human care" it is a goal that the nurses go into the investigation of this phenomenon whose purpose is to rescue the volues which characterize mankind collectives' culture and their specific contexts which have allowed them to solve health problems, and which are relevant to the care nature.

KEY WORDS: Nursing, holism, holistic, care, paradigm, hermeneutics, critical phenomenology.

\section{INTRODUCCIÓN}

En esta época de modernidad, caracterizada por una creciente crisis de valores y un proceso creciente de deshumanización en la atención a la salud de las personas, grupos y comunidades es innegable, la necesidad que existe en todos los campos del conocimiento, de encontrar respuestas a los problemas humanos. Es en este sentido que cobra relevancia el campo de las ciencias humanas y en particular de la filosofia, la que permitirá construir conocimientos desde un nuevo paradigma y nuevas racionalidades que fortalezcan la naturaleza de lo humano en la humanidad.

En la disciplina Enfermera es frecuente observar el asombro y el desconcierto que se experimenta, cuando se intenta hacer una aproximación sobre las bạses teóricas y filosóficas que sustentan el cuidado, -objeto de conocimiento de la Enfermería- debido a que predomina una concepción de profesión técnica, carente de autonomía y centrada más en el quehacer que en el ser; al respecto Silvina Malvárez. señala que "el devenir de oficio a profesión, de profesión a disciplina científica y de disciplina científica a ciencia y arte supone necesariamente un proceso histórico de racionalización del saber y de asumir una conciencia de si" ! Para fines del presente trabajo se parte de la noción de la Enfermería como ciencia y filoșofia del cuidado, refiriéndose al cuidado, bajo dos acepciones; una, como la relación de ayuda para la salud y la otra como el cuidado holístico, el cual promueve humanismo, salud y calidad de vida a la persona en su integralidad contextual.
El cuidado Holistico forma parte del metaparadigma* de Enfermería, en tanto que cuidado y junto con otros conceptos como el de persona, entorno y salud, constituyen los conceptos básicos filosóficos y epistémicos de la disciplina. Al cuidado holístico se le reconoce hoy más que nunca su valor ético que sustenta esta perspectiva tanto para la salud colectiva como para la salud de la Enfermera; persona humana en interacción permanente con la persona cuidada?

Este trabajo se centra en tres aspectos: Primero se define el holismo como un paradigma, sus origenes y su evolución, en seguida se vinculan estas concepciones con la Enfermerla como ciencia humanistica del cuidado y por último se analiza el cuidado holístico como fundamento para la práctica profesional.

\section{EL CONCEPTO DE HOLISMO. ORIGEN Y EVOLUCIÓN.}

Al holismo se le puede entender como una manera de ver los fenómenos en su totalidad, en su conjunto, en su complejidad, pues de esta forma es como se pueden apreciar las interacciones y sus particularidades en los procesos que por lo regular no se perciben si se estudian los aspectos que conforman el todo por separado.

El paradigma empírico analítico o positivista constituye un paradigma cientifico que ha permeado a muchas disciplinas, desde la Física, la Biologia, la Medicina, la Psicología y la Enfermería ${ }^{3}$, perspectiva que demuestra su. insuficiencia para estudiar y comprender los fenómenos 
humanos y sociales, surge asi un! nuevo paradigma, el fenomenológico, hermenéutico, interpretativo que acepta las contradicciones de la realidad, como son los fenómenos relativos a los valores, actitudes, creencias que caracterizan a la persona humana.

El holismo es la tendencia que permite entender los eventos desde el punto de vista de las múltiples interacciones, corresponde a una actitud integradora como también a una teorĺa explicativa que orienta hacia una comprensión contextual de los procesos, de los protagonistas y de sus contextos ${ }^{4}$

Etimológicamente deriva del vocablo griego "holos" se expresa en castellano como prefijo, hol u holo, y significa entero, completo, "todo"; indica también integro y organizado. Con holos se significa totalidad, relaciones, contexto o cualquier evento, aspecto, circunstancia, cualidad o cosa que en su momento esté siendo estudiado o tomado en cuenta, como "uno", como complejidad o como totalidads.

Aplicando el término en la investigación holística, el contexto, corresponde al holos de la misma. Holos, alude, entonces, a contextos, a complejidades, a realidades objeto de estudio, en relación con otros holos, es decir, con otros eventos, pues el holos es complejo, expresa múltiples relaciones, es dinámico y está en permanente relación ${ }^{6}$.

El holismo ha estado presente en el desarrollo del pensamiento univerșal. La comprensión filosófica del ser, desde sus inicios es holista, en la antigüedad fue representada con la escuela de Mileto, llamados Hilozoistas, los griegos más modernos no diferencian entre lo inanimado y animado, entre el espíritu y la materia; consideraban que todas las formas de vida eran manifestaciones de la físis, dotadas de vida y espiritualidad?

Desde la antigüedad el holismo ha estado presente en el desarrollo del pensamiento universal y ha influido de manera determinante en el campo de conocimiento de muchas ciencias.

La escuela de las totalidades significativas o Gestalt, escuela alemana surgida a finales del siglo XIX y principios del XX, en contraposición a la concepción atomista mecanicista, la cual reducía la comprensión de las experiencias en comportamientos más simples, estudiados por separados ${ }^{B}$.

Ya en el siglo XX el holismo se ve representado por el estadista sudafricano Jean Christian Smuts quien en 1926 publicó su libro "Holismo y Evolución" donde desarrolla

\section{HOLISMO Y SUS COMPONENTES}

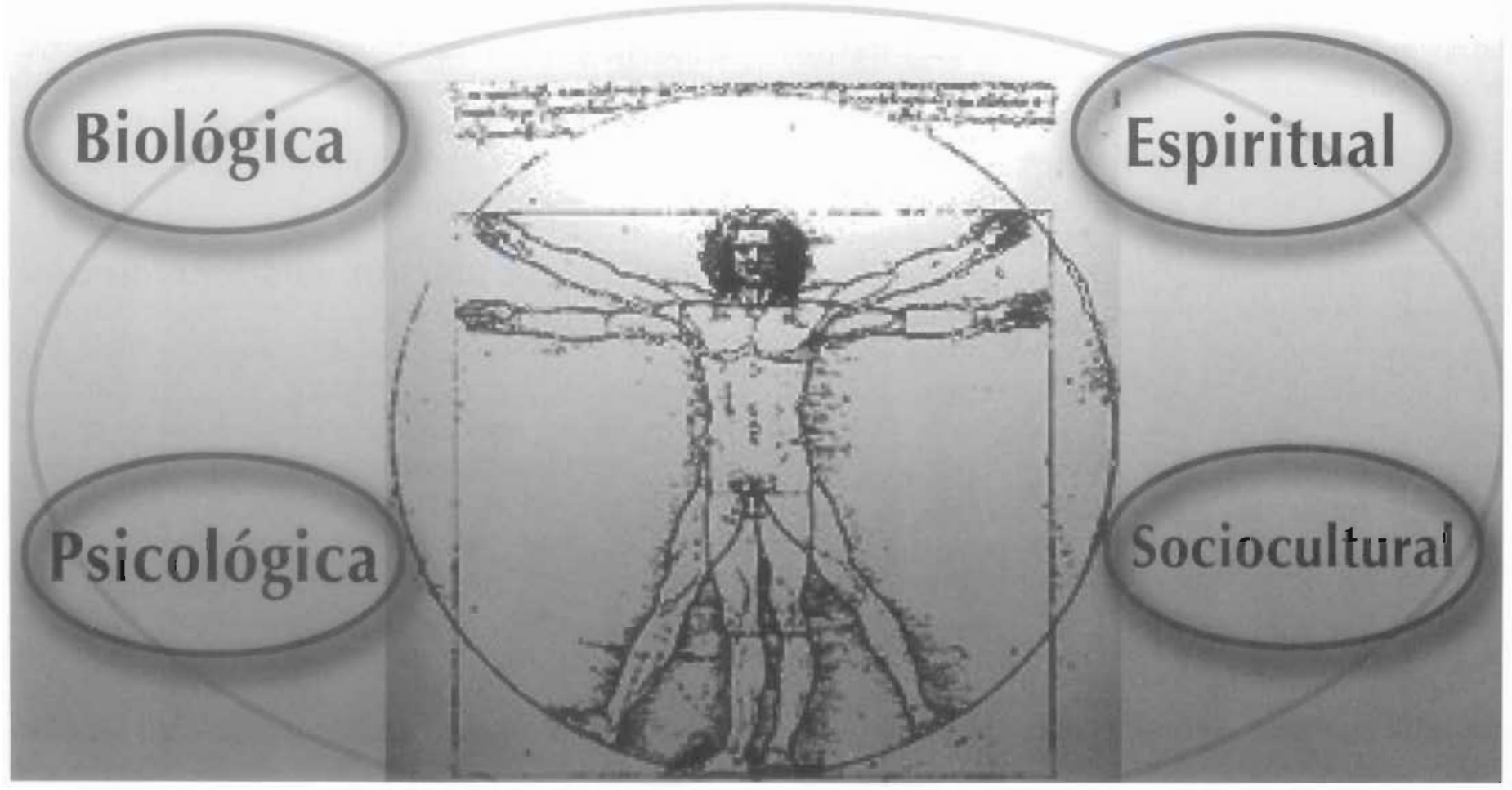


una concepción holística o de totalidad de las cosas según la cual, se configura el todo con sus partes, pero se observa que en la totalidad se producen realidades y efectos diferentes, a si se ven los productos por sus partes solamente. Kurt Goldstein, neuropsicólogo, considerado el primero en integrar el término holismo en las ciencias de la salud publicó en 1934 "El organismo. Una aproximación holística de la biologia", donde criticaba los dogmas de la neurología y la fisiología de la época, viendo al organismo como un sistema en el cual las estructuras cambiaban en interacción con el medio y con el mismo sistema, su tesis plantea que el organismo no puede ser dividido en "örganos" y menos en "mente" y "cuerpo", porque es el todo el que reacciona al ambiente. Establece un principio relativo a la comprensión de los fenómenos biológicos, en el cual propone que "las interacciones entre organismos, estímulos y órganos se dan con base en un proceso global armónico e integrado.".

En el área de la salud el holismo ha impactado de manera directa en la concepción de hombre, y en la interpretación de los fenómenos que explican la salud y enfermedad del mismo, tal es el caso que de prevalecer en un inicio la concepción biologicista la cual considera al hombre un ser funcional integrado por órganos y sistemas y a la salud y enfermedad como un proceso determinado directamente por funcionalidad orgánica; en la actualidad gracias a las aportaciones del holismo, el hombre es conșiderado como un ser total constituido por las dimensiones biopsicosocial, cultural y espiritual, todas ellas en intima y constante interacción entre sl y con su entorno global, abarcando incluso el cosmos, de tal mado que su salud o enfermedad dependen de su interacción continua, armónica y equilibrada ${ }^{10}$.

En la actualidad, el holismo șe ve revitalizado, pașa de ser un enfoque filosófico a constituirse como paradigma científico que está caracterizando a todos los campos disciplinares. La Enfermería como ciencia humana encuentra en el holismo un marco teórico congruente en șu fụndạmentaciọ́n.

\section{HOLISMO Y ENFERMERÍA}

El holismo en la Enfermería tiene una gran trascendencia ya que reafirma la dimensión humanistica del cuidado, en esta se reconoce al hombre como un ser total en el sentido de su determinación recíproca y por las múltiples interacciones con el entorno, la salud y el cuidado.

Cuando se aplica el concepto holístico a la persona y a la salud resalta el hecho de que las Enfermeras deberán respetar la identidad de "toda" la persona, y comprender, a la vez, la relación entre la parte del individuo que le precisa atender y la totalidad de las interrelaciones de la persona con él mismo y con su entorno ${ }^{11}$. En esta relación se reconoce al entorno físico, biológico, social y cultural que influencian y determinan dichas relaciones.

En la actualidad se viven situaciones de cuidado cada vez más complejas y obligan a una reflexión para la identificación de problemas y sobre todo para dar una respuesta. terapéutica a las necesidades de las personas en el proceso salud-enfermedad, por ello las intervenciones de Enfermería deberăn impactar de manera significativa y resolutiva en los niveles de salud real y potencial de la población, a partir de un conocimiento del comportamiento humano y del significado que tiene tanto la salud como la enfermedad para la persona que la padece y su familia, en este sentido es como el holismo fundamenta a la Enfermería.

El conocimiento de la disciplina está cada vez más influenciado por paradigmas interpretativos de la ciencia, donde el análisis del lenguaje juega un papel preponderante a través de la semántica y la semiótica de las manifestaciones culturales; asi mismo, identificar el significado que tiene "tener cuidado de una persona", lo que implica una transacción de cuidados profesionales, personales, científicos, estéticos, humanos y éticos, expresados en un todo integrado (holístico), que involucra un proceso de conocimiento y de acción de la Enfermera en los cuidados culturales.

Leininger $^{12}$, aporta a través de su teoría de los cuidados transculturales supuestos que carạcterizan las prácticas de cuidado bajo un enfoque holístico, superando de manera significativa la visión fragmentada de la persona, el cuidado, la salud y el entorno que otras teoristas han planteado.

Es una aspiración filosófica, ética, y estética de la Enfermería que en la consideración de disciplina humana, se consolide el paradigma holístico para concretarse en su práctica.

\section{EL CUIDADO HOLÍSTICO}

Hablar de cuidado holistico es reconocer que las experiencias de vida del ser humano son punto de partida para explicar sus comportamientos como seres individuales, aceptando su dimensión subjetiva e intersubjetiva, su experiencia, su creatividad y desarrollo.

El cuidado holística se centra en la ayuda a la persona, la familia y los grupos para hacer frente a los cambios y a las crisis de una manera adaptativa; potenciar sus 
recursos y experiencias como un elemento de crecimiento en beneficio de su salud.

El cuidado holístico es además promotor de salud, ya que concibe a la persona capaz de construir una nueva estructura mental que le permita tomar decisiones en pro de la satisfacción de "sus" necesidades sin poner en riesgo su bienestar futuro; asi mismo es un enfoque de crecimiento y sanación integral, en lo individual y en lo colectivo, facilitando el desarrollo del potencial vital y creativo más profundo de la persona cuidada y de quien brinda este cuidado ${ }^{13}$.

La Enfermeria posee un corpus de conocimientos multidisciplinar que sustentan el holismo, expresados en teorias y modelos que le permiten entender los mecanismos que determinan y condicionan los procesos de salud de la persona y la posibilitan a otorgar cuidado holístico, como ejemplo de teorias con este enfoque está entre otras: Filosofía y Ciencia del Cuidado de Jean Watson, que ofrece un planteamiento claro sobre la naturaleza del cuidado humano, establece los fundamentos para el ejercicio de un rol autónomo, plantea cuestiones sobre la naturaleza de los saberes fundamentales y los conceptos que deberán ser la matriz conceptual para ofrecer cuidados enfermeros profesionales, invita a aproximar los cuidados con una visión centrada en la salud en la que la dimensión biológica psicológica, social, cultural y el entorno de la persona es tomada en cuenta en sus interacciones.

Otro ejemplo es la perspectiva teórica de Las Respuestas Humanas; ante la salud y la enfermedad, esta explica las reacciones específicas de cada persona cuando vive un problema de salud o a una crisis del desarrollo, la Enfermería vincula este referente con el contexto socio cultural de la persona y le da un significado particular; que le permitirá implementar estrategias únicas e individualizadas para la resolución de problemas en situaciones de: estrés, de dolor, de perdidas, del nacimiento, de la lactancia, de la vejez etc,

El cuidado holístico constituye en si un enfoque paradigmático que deberá ser valorado, estudiado y aplicado en la práctica tanto a nivel institucional como comunitario, de manera que se vea transformado el actual sistema de cuidados y asi lograr elevar el prestigio y autonomía de la profesión.

Al ser definida la Enfermería como ciencia humana de experiencias de salud-enfermedad-curación, que se expresa a través de cuidados humanos, personales, científicos, éticos y estéticos: requiere que sus profesionales sean formados bajo esta orientación, de manera que incorpore prácticas alternativas para el cuidado vinculadas con las creencias y valores de las poblaciones, pero sobre todo con formación humanistica: ética y estética que le permita valorar el respeto a la individualidad, a la integridad de la persona, los derechos humanos y a desarrollar habilidades para el autoconocimiento, autocontrol y autocuidado.

Siguiendo a Husser! en su definición de las ciencias humanas, donde el interés de estudio es el ser humano: como persona, su vida personal, su actividad, etc., interesa también conocer el como vive la persona en su mundo social, en el que el yo y el nosotros viven en comunidad y tienen a la comunidad como horizonte. Aquí la palabra vida es tomada como creación cultural en una continuidad histórica ${ }^{14}$. Según esta aportación el cuidado holístico puede ser estudiado como actividad humana tal cual es vivida por las personas.

Los practicantes de las ciencias humanas deben considerar el espiritu como parte esencial de la persona en y con sus fundamentos corporales, por ello la Enfermera holistica se funda en un sistema de valores orientados hacia las siguientes factores cuidativos que Watson propone para el proceso de cuidados cooparticipantes, cada uno de ellos tiene un componente fenomenológico que es pertinente a las personas implicadas en la relación que genera la Enfermeria, estos son:

- La formación de un sistema de valores humanísticos y altruistas; En donde la Enfermería ejerce una influencia en los valores y experimenta la satisfacción de dar y recrear el significado de persona.

- Fomenta la fe y la esperanza; En el sentido de la promoción del bienestar, al ayudar a la persona a adoptar conductas saludables, gestionar apoyo y lograr una eficaz relación Enfermera-persona.

- Cultivar la sensibilidad hacia uno mismo y de los demás; En donde se conduce a la autorrealización a través de la autoaceptación tanto de la Enfermera como de la persona.

- Desarrollar una relación de cuidados de ayuda y confianza; Tiene la finalidad de promover $y$ aceptar sentimientos positivos y negativos para una comunicación efectiva.

- Promocionar y aceptar la expresión de sentimientos positivos y negativos; En el sentido de ser capaz de entender que la interpretación intelectual es distinta a la interpretación emocional

- Usar métodos en la resolución de problemas; Se hace referencia al uso del método de Enfermería para el cuidado. 
- Promocionar la enseñanza-aprendizaje interpersonal; En éste factor se trasciende del cuidado curativo al autocuidado a la salud en donde la enfermera informa y traslada la responsabilidad del bienestar y la salud de su persona al propio paciente.

- Crear un entorno de apoyo o sanación mental, física, sociocultural y espiritual; Aqui el rol de la Enfermera es reconocer la influencia del entorno interno y externo de la salud-enfermedad de las personas.

- Ayudar en la satisfacción de las necesidades humanas; Se entiende que la Enfermera reconoce la existencia de necesidades biofísicas, psicofísicas, psicosociales e interpersonales de si misma y del paciente.

- Aceptación de fuerzas existenciales fenomenológicas; En donde se reconoce que la fenomenología describe los datos de la situación inmediata que ayuda a las personas a comprender el fenómeno en cuestión.

Esta perspectiva de Watson permite articular una particularidad de los cuidados enfermeros con una característica de identidad como lo es la consideración holística de la naturaleza humana ${ }^{15}$.

Lo anterior nos lleva necesariamente a reconocer que los fenómenos del Cuidado, tendrán que ser estudiados como totalidades, producto del conjunto de relaciones, las cuales corresponden a eventos sucedidos, a otros que se suceden ya un infinito número que están por sucederse.

A manera de conclusión se puede decir que el cuidado holístico, proyecta a la Enfermera** como profesional autónomo, con capacidad para resolver problemas reales de salud en el proceso vital humano, en el desarrollo personal y de crisisi a nivel individual, familiar y de grupos, la posibilita a incursionar y dominar prácticas de cuidados alternativos culturalmente valiosos para la población, le permite controlar y promover entornos saludables, proporcionar confort espiritual y con ello garantizar la seguridad y salud de la persona en su integralidad.

\section{REFERENCIAS BIBLIOGRÁFICAS}

I Malvárez, S. Fundamentos en la construcción del conocimiento en Enfermeria, filosófico y ético. En: Desarrollo cientifico de Enfermería, Vol. 10, No. 8, septembre 2002, pp. 233-239

2 Watson, 1. Nursing: The philosophy and science of caring. Boston: Little, Brown. 1979.

3 Fritjof, Capra. El Tao de la Fișica. Ed. Sirio, Barcelona, 1997. p. 199
4 Arroyo, M.P. en "Cuidado de Enfermería, una reflexión desde la práctica Hospitalaria" http//wwwencolombia.com, medicina/enfermería.

5 Santillana . Diccionario de las ciencias de la Educación. Ed. Santillana, México, 1995. p. 734-735.

6 Ibidem. P. 736-737

7 Fritjof, Capra. El Tọ de la Fisica. Ed. Sirio, Barcelona, 1997. p. 199

8 Kozier, B. et.al. Fundamentos de Enfermeria. Ed. Interamericana, México, 1984, p.72

9 Verstegen.M.Ivan, "Mandelbaum as a Gestalt Philosopher. Http://www.geocities.com/hostpring/Maurice 10 Krieger, D. Fundations for holistic health nursing practices. Ed. Lippincot Philadelphia, 1981, p.4.

11 Marriner-Tommey et al. Modelos y Teorias de Enfermería. Ed Roll. Barcelona, 1989. pp. 125-135.

12 Leininger, M. Trancultural Nursing: Concept, theorist and practices. New Cork: Jhon Wiley\& Sons. 1978. pp. 12-15.

13 Watson, J. Nursing: Human sciencie and Human CARE: a Theory of Nursing. New York: National League for Nursing Press. 1988. pp. 28

14 Husserl, E. Phenomenology and crisis of the philosophy. Ed. Harper and Row. New York, 1995. p. 150

15 Medina, J. La Pedagogía del Cuidado: Saberes y prácticas en la Formación Universitaria de Enfermería. Ed. Alertes, Barcelona, 1999. p. 49-53.

\section{NOTAS}

* Para Carmen Fernández Ferrin y cols. (1997)

El metaparadigma, es la perspectiva más global de una disciplina, este identifica los fenómenos propios de la disciplina, y la perspectiva general adoptada para observarlos.

** El término Enfermera se empleará para hacer mención tanto al género femenino y masculino.

\section{DIRECCIÓN PARA CORRESPONDENCIA}

Sofia Rodriguez Jiménez: eneo_sofi@hotmail.com

Sandra. Sotomayor Sánchez: sootomayor1999@yahoo.com.mx

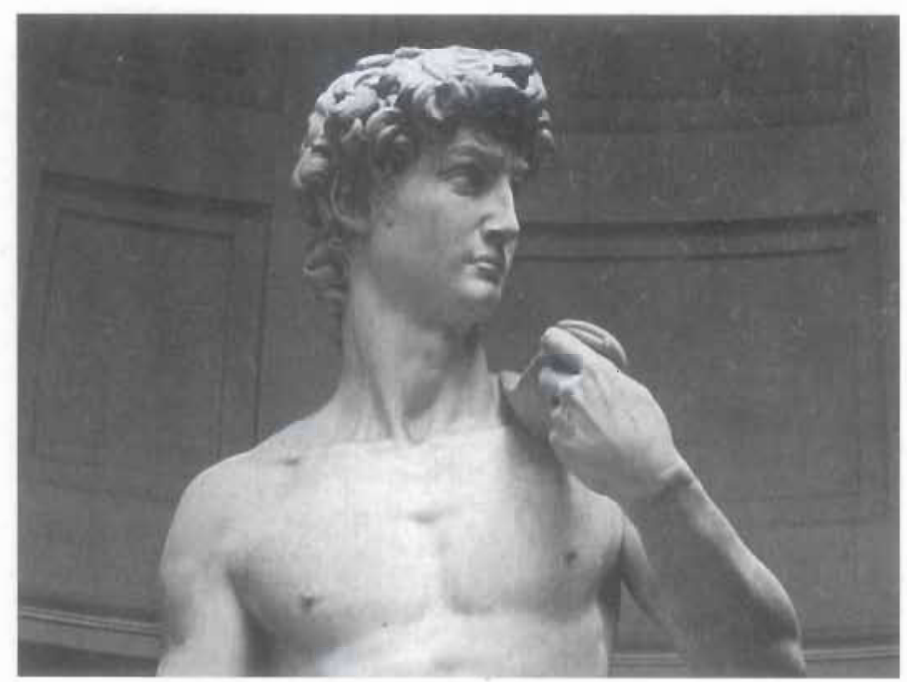

